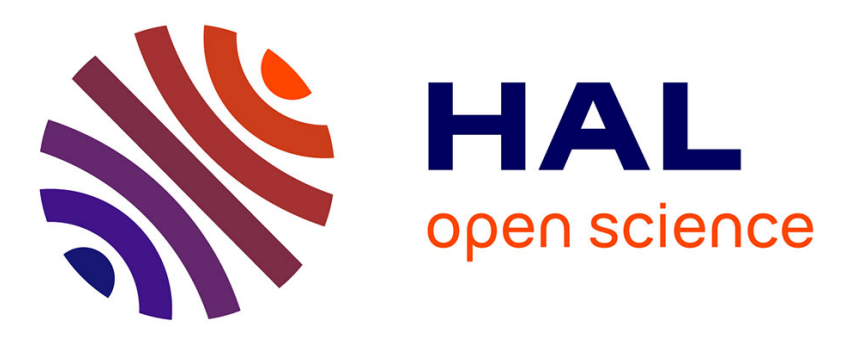

\title{
Aquatis and Ryujin projects: First steps to remote sensing bottom of the sea by small homemade Autonomous Underwater Vehicles
}

Laurent Beaudoin, Loïca Avanthey, Antoine Gademer, Vincent Vittori, Laurent Dupessey, Jean-Paul Rudant

\section{To cite this version:}

Laurent Beaudoin, Loïca Avanthey, Antoine Gademer, Vincent Vittori, Laurent Dupessey, et al.. Aquatis and Ryujin projects: First steps to remote sensing bottom of the sea by small homemade Autonomous Underwater Vehicles. IEEE International Geoscience and Remote Sensing Symposium (IGARSS), 2012, Munich, Germany. 10.1109/IGARSS.2012.6352405 · hal-01132238

\section{HAL Id: hal-01132238 \\ https://hal.science/hal-01132238}

Submitted on 16 Mar 2015

HAL is a multi-disciplinary open access archive for the deposit and dissemination of scientific research documents, whether they are published or not. The documents may come from teaching and research institutions in France or abroad, or from public or private research centers.
L'archive ouverte pluridisciplinaire HAL, est destinée au dépôt et à la diffusion de documents scientifiques de niveau recherche, publiés ou non, émanant des établissements d'enseignement et de recherche français ou étrangers, des laboratoires publics ou privés. 


\section{AQUATIS AND RYUJIN PROJECTS: FIRST STEPS TO REMOTE SENSING OF THE SEA BOTTOM BY SMALL HOMEMADE AUTONOMOUS UNDERWATER VEHICLES}

\author{
L. Beaudoin, L. Avanthey, A. Gademer, \\ V. Vittori, L. Dupessey* \\ ESIEA \\ Laboratoire ATIS \\ 9 rue Vésale, F-75005 Paris, France
}

\author{
J.-P. Rudant \\ Université de Marne-la-Vallée, \\ GT / IFSA \\ Laboratoire ESYCOM, \\ Cellule GT (Géomatique, Télédétection) \\ 5 Bd Descartes, F-77454 Marne-la-Vallée, France
}

\section{INTRODUCTION}

For many applications such as pipeline surveys, environmental, archeological or security studies, precise information on the sea bottom can be critical. But because of the difficulties of underwater exploration, even coastal areas are mostly unsurveyed. The main issues are due to the hostility of the sea environment: pressure, salinity, and a limited visibility (less than 15 meters most of the time). It is also very difficult to get a precise localisation: there is a huge drift and it is impossible to use such device as the GPS data for live geo-positionning. For all these reasons, it takes a lot of time to cover an area undersea, and the use of divers may be too dangerous or expensive. That is why the use of underwater robots is considered as an efficient solution: it allows deeper and longer diving as well as the use of optimized payloads.

In a divide and conquer approach, using a team of robots allows to decrease the time needed to cover the area of interest. The main hindrance to such development is the cost ( $>10,000$ euros) and the size (manipulation, transportation, etc.) of the robots. That is why we present in this article two small low cost homemade underwater robots. These robots are called Aquatis and Ryujin and benefited from the experience earned on our previous aerial robotic work [?, ?, ?]. A first section summarize the technical aspects of the robots (mechanical and electronic designs, software developments). Then we present the first operational results obtained during pool tests and also during an international student contest dedicated to autonomous underwater robotics. This section ends with pedagogical return of experience.

\section{OVERVIEW OF THE ROBOTS}

\subsection{Two robots, two approaches}

The AQUATIS robot is quite conventional in its structure. Its goal is to educate the students in how to build an underwater vehicle (about 8,000 euros) and to increase their awareness of the harsh conditions of this environment. This being done, they can rapidly learn the basis of programming Autonomous Underwater Vehicles (AUVs). Then they can concentrate on high level development and try to innovate in this area. The robot is dedicated to perform automated mission.

When we discovered the SeaPerch [?] in the summer of 2010, we decided to do some research on an advanced design. It gave birth to the RYUJIN robot that stands as an alternative to the AQUATIS AUV. Its structure is radically different from

\footnotetext{
*Thanks to Fischer connectors Society for sponsoring us and Charenton-le-Pont city for diving and simming pools access.
} 


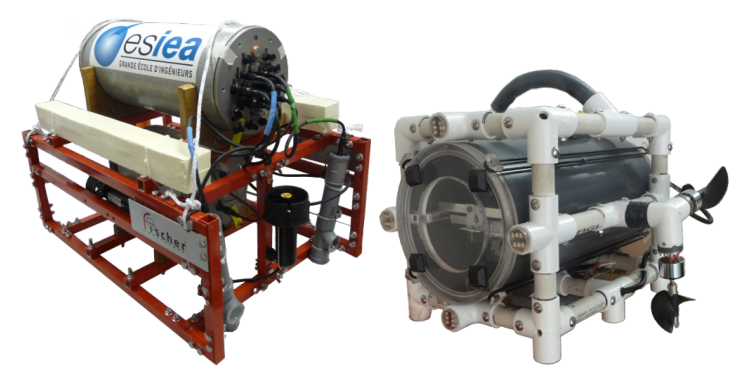

Fig. 1. The AQUATIS (left) and RYUJIN (right) robots.

robots of the same category. Its key features: small size, easy to build and low cost (about 1,000 euros). Its goals: to achieve automated missions with the same efficiency as the other robots and serve as a learning platform for students working in the laboratory.

Designing an underwater robot implies combining knowledge in various domains but we can group them under three main functions: sealing the hull, connecting sensors and electronics, and low and high level algorithmics.

\subsection{Hardware and mechanics}

\subsubsection{AQUATIS}

Basically, the hull of the AQUATIS robot is mainly composed of two aluminum alloy tubes of $25 \mathrm{~cm}$ and $55 \mathrm{~cm}$ long and 21 $\mathrm{cm}$ in diameter and an external cubic frame. The long component contains all the embedded hardware and the other the NiMH batteries. So, it is relatively easy to replace flat batteries without opening the robot for long time mission. The purpose of two separate compartments is to prevent interference from the IMU.The external frame serves to hold the tubes, and thrusters; and carry all sensors. The movements of the robot are performed by four thrusters, two on vertical and two on horizontal planes. For that we use BTD 150 thrusters from Seabotix associated with Graupner Navy V15R speed controllers. The overall structure is $75 \mathrm{~cm}$ long, $51 \mathrm{~cm}$ wide and $57 \mathrm{~cm}$ height (figure ??, left), for $39 \mathrm{~kg}$ weight [?].

\subsubsection{RYUJIN}

First of all, the mechanic parts were assembled with the simplest tools we could use such as saws, drilling machine, screw drivers and a CNC machine for the caps. The robot is made of a unique tubular housing in PVC where the electronic is housed. It is surrounded by a submerged frame of PVC pipe that maintains it as well as the sensors and the motors. This has many advantages. On the one hand, the low cost of PVC makes the material very accessible, which fits very well with our desire to design a low-cost robot. On the other hand it allows multiple assemblies which gives us the opportunity to build the form we want and change it at will. The small size of the robot makes it easier to test and to transport.

The main challenge in designing this robot was to overcome the technical hindrance of the propulsion system. Most underwater propulsion systems on the shelf are relatively big (more than $10 \mathrm{~cm}$ long), which dictates the size of the robot. Therefore we chose to modify small motors in order that they can run in salt water and under pressure. It was quite a bet but it works very well. The final fully-equipped robot weighs under $10 \mathrm{Kg}$ and measures $30 \mathrm{~cm}$ x $20 \mathrm{~cm}$ x $20 \mathrm{~cm}$ (figure ??, right). 


\subsection{Electronics design and Sensors}

\subsubsection{AQUATIS}

The brain of the robot is an embedded computer. The communication with the different sensors is done via a pyramidal architecture. On the top, all the entries of the computer are in USB mode. Some sensors are USB native, but others not. So, the next level of the pyramid is dedicated to the conversion of different communication and control protocols in USB. To do that, we use a Controller Area Network or CAN that is connected to homemade multifunction boards. The gateway between the high level computer and the multifunction boards is an Ethernet/CAN module. On the other, different protocols (analog, RS232, RS485, PWM) are converted into CAN signal. These conversions are done by the same homemade board which reduces logistic constraints. The CAN allows use giving each item of data an id, and priority handling.

\subsubsection{RYUJIN}

The electronics of the RYUJIN robot had to be as small as possible in order to fit in the small housing. As we had gained experience on working with UAVs in the laboratory (it is one of our lines of research), we designed electronics that not only fits the recommendation for the application but also our learning approach for our students. Indeed, it is very similar to our other projects, which are all based on our homemade learning card, called ULC. This way, students can jump from one project to another, either aerial or underwater, without many changes in the electronics. Moreover, each enhancement on either of the projects can be transferred to the other ones. We have built a frame that slides out of the pipe when you want to reach the electronic cards. This frame is fixed to the rear plug with 4 screws and allows us to plug on it the following devices: the power supply board, the 4 electronic drives, the batteries, the sensor card, the motherboard and the internal camera.

\subsubsection{Sensors}

We put in both robots the conventional sensors like temperature and pressure sensors to compute the depth at which the robot is navigating, and even a humidity sensor to detect leaks inside the watertight chamber. Both robots have IMU to estimate their attitude To help navigation and collect data we also put a Sonar on the AQUATIS robot and underwater video cameras with spotlights on both. We are currently developing some applications and algorithms with video and sonar put together.

\section{FIRST RESULTS}

\subsection{Pool tests}

The first tests have been made in a diving pool (upto $15 \mathrm{~m}$ deep) to validate the waterproofness of the structures and sensors. Then, we developed command, control, auto-piloting and vision algorithms in a swimming pool. This kind of environment is particularly favorable: very clear water, about 30 degree celsius temperature, no waves and no current. Obviously, in real operational environments, the problem is much more complicated. The first campaigns in sea water were made during the student contest called SAUC-E.

\subsection{The SAUC-E contest}

The Student Autonomous Underwater Vehicle Challenge Europe (SAUC-E) aims to stimulate innovation and technology, and encourage young people to pursue careers in engineering and underwater related areas. The event was organized by the NATO Undersea Research Centre (NURC) in La Spezia (Italy). Students were asked to design and build AUVs capable of performing real missions tasks like following a prescribed route, finding and passing through validation gates, finding and following a 
pipeline, surveying a wall of a dock, finding a mid-water target and cutting the rope that fixes it to the bottom, performing tracking of a moving surface target and going to a predetermined surfacing zone.

It is the second year that we have participated in this contest. In 2010, we presented the Aquatis robot, after one academic year of work from the student team, and in 2011, we presented the newly created RYUJIN robot at AQUATISs side. This year, we managed to participate in the final run with the RYUJIN AUV and ranked 5th in the contest. Unfortunately, for some mechanical problems, AQUATIS could not make it in time for the qualification runs. The ESIEA robots received two awards "Best use of resources" award for the team RYUJIN and "Perseverance and tenacity" award to the team AQUATIS.

\section{PEDAGOGICAL ASPECTS}

These two research projects are closely related to the work of students in our engineering school. It is a will of our laboratory to encourage the creativity of our students and to work within the constraints imposed by their workloads. In fact for most of those who participate in these research programs, their work on these projects is graded. Thus they participate in some pieces of the UAV to make it more competitive or efficient in a predefined task.

We accept students from the second year of their course upto PhD students. Concretely, the 2011 AQUATIS students team were between their second and fourth year of studies and the RYUJINs team was essentially composed of students between fourth year and $\mathrm{PhD}$. That experience allows them to see what the research world is like and it gives them the opportunity to work in challenging and innovating projects in collaboration with researchers.

\section{CONCLUSION}

We have presented in this article two research and pedagogical projects which aim to develop low-cost light AUV for collaborative mapping. Both are designed for operational missions and teaching of computer science and electronics with a focus on localisation sensors for AQUATIS and advanced design for RYUJIN. We plan to use a sonar coupled with video to build visual odometry and be able to make Simultaneous Localisation And Mapping (SLAM) with 3D reconstruction of the scene the robots are moving in.

Besides, on our work on the UAV, we use LIDAR sensors. We want to test them in an underwater environment to compare the acquired data with those of the Sonar and to get more information of distance and environment. Eventually, we want to be able to merge those data with the other information we already have from the other sensors.

Finally, we plan to multiply the number of AUVs to be more prepared at an operational point of view and also to develop swarms of cheap AUVs in order to have more efficient mapping and work for other purpose like rescue, surveying, etc. 